\title{
Self-Assembly of Polyhedral Hybrid Colloidal Particles
}

\section{Citation}

Perro, Adeline, Etienne Duguet, Serge Ravaine, and Vinothan N. Manoharan. 2008. Self-

Assembly of polyhedral hybrid colloidal particles. MRS Online Proceedings 1135: 1135-CC06-08.

\section{Published Version}

doi:10.1557/PROC-1135-CC06-08

\section{Permanent link}

http://nrs.harvard.edu/urn-3:HUL.InstRepos:8000958

\section{Terms of Use}

This article was downloaded from Harvard University's DASH repository, and is made available under the terms and conditions applicable to Other Posted Material, as set forth at http:// nrs.harvard.edu/urn-3:HUL.InstRepos:dash.current.terms-of-use\#LAA

\section{Share Your Story}

The Harvard community has made this article openly available.

Please share how this access benefits you. Submit a story.

Accessibility 


\title{
Self-Assembly of Polyhedral Hybrid Colloidal Particles
}

\author{
Adeline Perro $^{1}$, Etienne Duguet ${ }^{3}$, Serge Ravaine ${ }^{4}$ and Vinothan N. Manoharan ${ }^{1,2}$ \\ ${ }^{1}$ School of Engineering and Applied Sciences, Harvard University, Cambridge MA 02138, \\ USA, \\ ${ }^{2}$ Department of Physics, Harvard University, Cambridge MA 02138, USA, \\ ${ }^{3}$ CNRS, Université de Bordeaux, ICMCB, Pessac, France, \\ ${ }^{4}$ CNRS, Université de Bordeaux, CRPP, Pessac, France.
}

\begin{abstract}
We have developed a new method to produce hybrid particles with polyhedral shapes in very high yield (liter quantities at up to $70 \%$ purity) using a combination of emulsion polymerization and inorganic surface chemistry. The procedure has been generalized to create complex geometries, including hybrid line segments, triangles, tetrahedra, octahedra, and more. The optical properties of these particles are tailored for studying their dynamics and self-assembly. For example, we produce systems that consist of index-matched spheres allowing us to define the position of each elementary particle in three-dimensional space. We present some preliminary studies on the self-assembly of these complex shaped systems based on electron and optical microscopy.
\end{abstract}

\section{INTRODUCTION}

Non-spherical colloids are potential precursors for new materials such as 3D photonic crystals with a band gap in the visible spectrum. Here we describe a seeded emulsion polymerization technique to create such particles. The main advantage of this technique over other techniques that use emulsion droplets as templates [1] is that it is allows for a much higher yield of specific types of clusters. Also, we show that it is possible to create some colloidal particles composed of crosslinked polymer that can be index matched in an appropriate solvent. Moreover, studying the crystallization of these colloids could yield insights into new methods of making photonic crystals.

\section{EXPERIMENTAL DETAILS}

Materials. Tetraethoxysilane (TEOS, 99\%, Sigma), styrene (99\%, Alfa Aesar), ethylene glycol dimethacrylate (EGDMA, TLC), sodium persulfate (98\%, Sigma), and ammonia (25\% in water, SDS) were purchased as reagent grade and used without further purification. Methyl methacrylate (MMA, 99\%, Alfa Aesar) was purified by distillation before use. Methacryloxymethyltriethoxysilane (MMS) were purchased from Gelest, the surfactant Synperonic NP30 from Fluka and Norland 68 optical adhesive from Norland. Water was Milli-Q grade (Millipore).

Synthetic Procedures. Silica seeds were synthesized using a procedure described elsewhere.[2] To favor the growth of the polymer beads onto the mineral surface, silica particles were surfaced modified by adding MMS at a concentration that yielded 1 functional group per $\mathrm{nm}^{2} .50 \mathrm{~mL}$ of surface modified silica suspension were introduced in a three-neck round flask of $250 \mathrm{~mL}$ 
equipped with a chilled condenser. The mixture was stirred at $300 \mathrm{rpm}$ under nitrogen for 2 hours. $150 \mathrm{mg}$ of surfactant (Synperonic NP30), $5 \mathrm{~g}$ of monomer (styrene or MMA + EGDMA $(15 \% \mathrm{w} / \mathrm{w}))$ were then introduced. The mixture was heated at $70^{\circ} \mathrm{C}$ before the introduction of 25 mg of sodium persulfate. Polymerization was carried out for 2 hours under magnetic stirring. Crystals were made through slow sedimentation.[3] PMMA-co-EGDMA latexes at 2\% volume fraction were placed in a mixture consisting of $75 \%$ toluene, $25 \%$ absolute ethanol (\% vol.), and $1 \times 10^{-4} \mathrm{M}$ of Tetra-n-butylammonium chloride salt. Then this solution was introduced into a 100 $\mu \mathrm{m}$ cell fabricated with a coverslip deposited on a glass slide separated by Dupont Teijin Films and sealed with optical adhesive to prevent the suspension from evaporating.

\section{DISCUSSION}

As shown in Figure 1A, we obtained various polyhedral hybrid colloids composed of a silica core surrounded by a precise number of polystyrene beads.
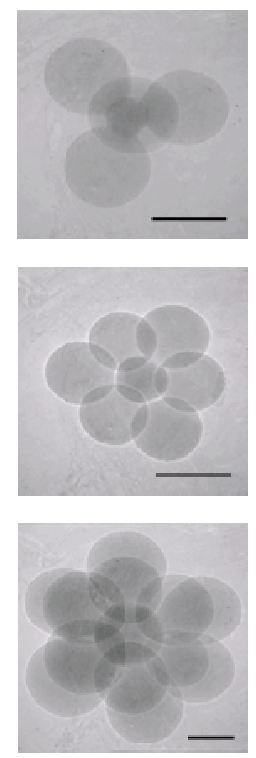

$200 \mathrm{~nm}$
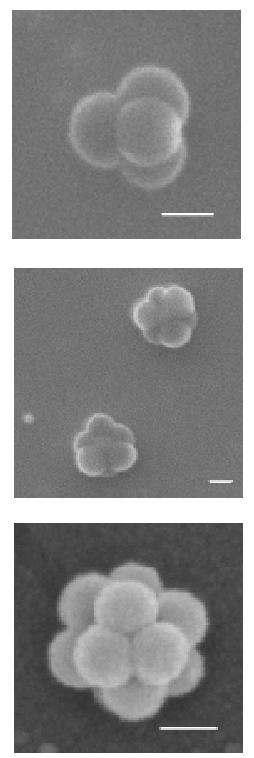

$200 \mathrm{~nm}$

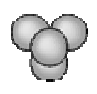

A

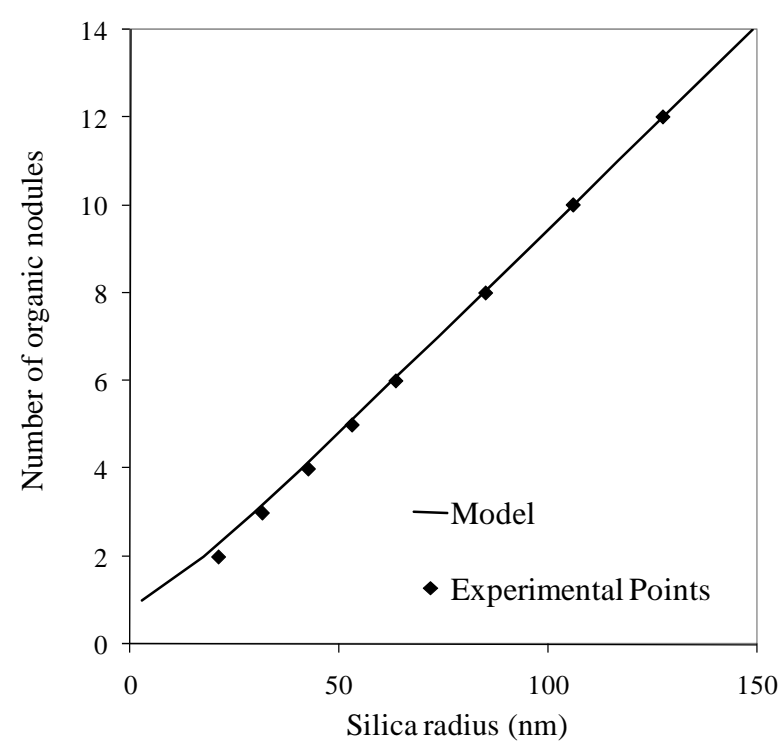

B

Figure 1: A) Scanning and transmission electron microscopy pictures of binary clusters obtained after 2 hours with silica seeds of different diameters. B) Evolution of number of growing polystyrene as a function of the silica seeds size.

As shown in prior work by Perro et al.,[4] the morphology of the colloidal clusters can be modeled using hard-sphere geometry. Mathematically this model is equivalent to minimization of a two-term-energy. The first term represents an attraction towards the center, while the second term represents hard particle repulsion. Figure $1 \mathrm{~B}$ shows the evolution of the silica particle radius $\mathrm{R}_{\mathrm{Si}}$ as a function of $\mathrm{N}_{\mathrm{PS} / \mathrm{Si}}$ observed experimentally and fitted using the following equation: 


$$
R_{S i}=K\left(\frac{2 N_{P S / S i}}{3}-\frac{1}{2 N_{P S / S i}}\right)
$$

where $R_{S i}$ is the silica seed radius, $N_{P S / S i}$, is the number of polystyrene beads growing on each silica particles, and $\mathrm{K}$ is an arbitrary constant.

Figure $1 \mathrm{~B}$ shows that this equation yields a good fit to the data. Note that our model does not explain the physical mechanism for the formation of the clusters; it only shows that the cluster morphology follows a straightforward geometric rule. We use the fitted data to predict what morphologies will form under constant reaction conditions for a given silica sphere size.

In order to study the formation of colloidal crystals of the polyhedral clusters, we attempted to extend the synthesis procedure to PMMA-co-EGDMA particles instead of polystyrene. The resulting colloidal suspension could be easily index matched in toluene as depicted on Figure 2 . We note that in addition to the transparency of the dispersion, a dominant blue color induced by Rayleigh-Gans scattering is visible in transmission. The role of the EGDMA is to crosslink the particles, allowing them to be swollen by the solvent without dissolution. To prepare polyhedral clusters of a given morphology, we used our model, fitted using the data for polystyrene, as a guide.

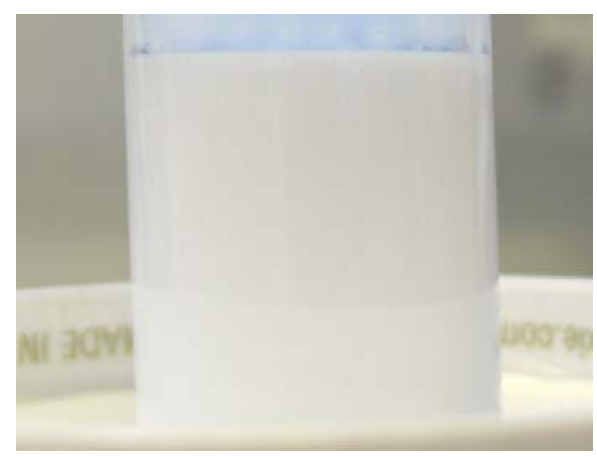

A

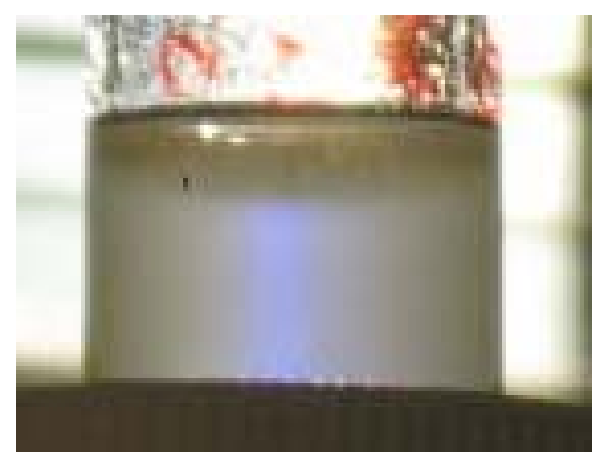

B

Figure 2: Images of PMMA-co-EGDMA latexes in A) water, B) Toluene.

In the first experiment, a colloidal crystal composed of only spherical PMMA-co-EGDMA particles was prepared through sedimentation (see Methods section). No silica particles were used in the preparation of the polymer spheres. Figure 3 A shows Bragg diffraction due to the formation of crystals in the bottom of the cell. Optical microscopy (Figures 3 B) reveals the presence of several crystallites about 20 microns in diameter with face centered cubic morphology (Figure 3C) as expected. 


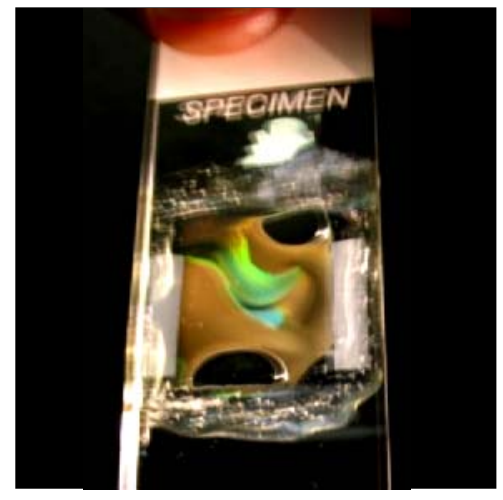

A

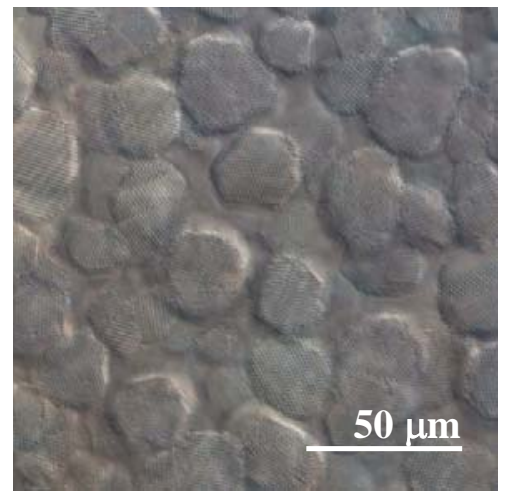

B

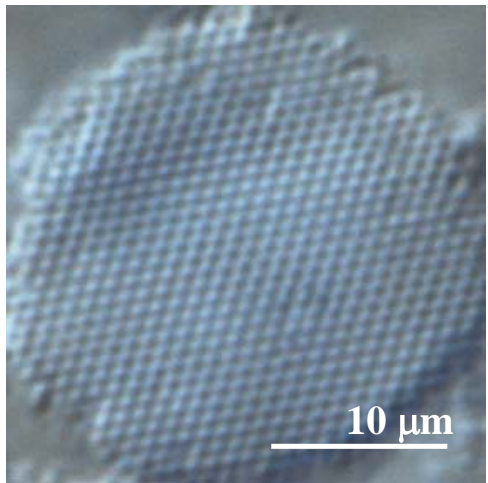

$\mathrm{C}$

Figure 3: A) Macroscopic image of a photonic crystal composed of spherical PMMA-coEGDMA particles, B) Optical micrograph of crystallites, C) Focus on one crystallite.

In a second experiment, we studied the crystallization of a suspension containing both dimers and spheres. $42 \mathrm{~nm}$ silica seeds were introduced in the polymerization media, yielding a solution containing 15\% dimers by number (Figure 4A). This was a fairly low yield of dimers, compared to the same synthesis in polystyrene. The difference in yield might be due to the difference in polymerization kinetics between polystyrene and PMMA.

We prepared crystals of the dimer mixture using the same method as above. After a few days blue crystals appeared in the cell, indicating the formation of crystallites. Optical microscopic pictures (Figure 4B) show that the crystallites were cauliflower-shaped. A closer look at picture $\mathrm{C}$ shows the presence of dislocation lines centered around one central point. It is possible that these defects could be attributed to the presence of dimers.[5] With our current technique it is not possible to distinguish between dimers and single spheres in the crystals, but future work might involve fluorescently labeling the silica sphere so that the dimers can be located.

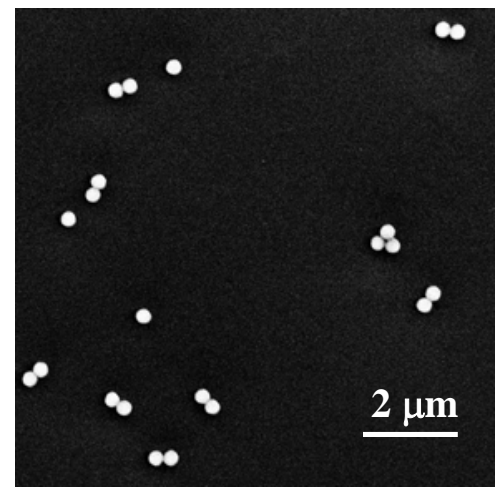

A

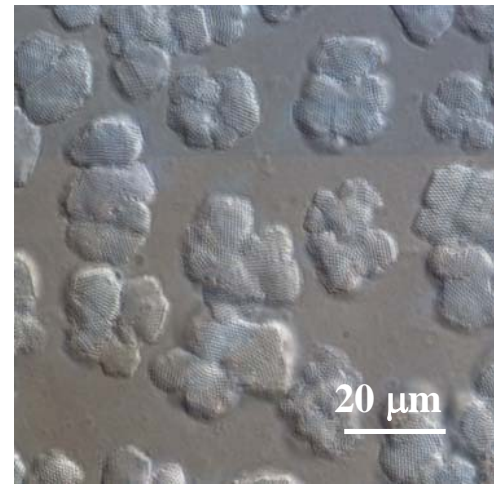

B

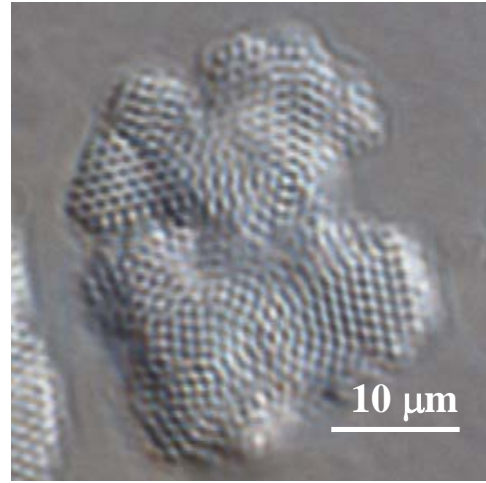

C

Figure 4: A) Scanning electron microscopic image of PMMA-co-EGDMA dimer suspension (dried), showing the presence of single spheres and trimers in addition to dimers, which were $15 \%$ by number, B) Image of a photonic crystal composed of a solution containing $15 \%$ of hybrid dimers (Silica / PMMA-co-EGDMA spheres), C) Focus on one crystallite. 


\section{CONCLUSION}

We have demonstrated the fabrication of regular polyhedral hybrid colloids that are stable in toluene. Single spheres and dimers assemble into colloidal crystals that can be studied using optical microscopy; the crystallite morphology varies with the fraction of dimers. Because the particles are nearly index-matched, confocal microscopy could be used to study the structure of the crystallites. Indeed, introducing fluorescent dye in the continuous media should allow us to study how these crystallites are formed.[6] Future work will involve studying the effects of increasing the dimer concentration on the crystallite morphology. Also we plan to optimize the fraction of a given morphology by varying the reaction conditions. This may allow us to selfassemble colloidal crystals consisting of, for example, pure dimers or pure tetrahedra.

\section{ACKNOWLEDGMENTS}

This research is financially supported by Egide (Lavoisier Fellowship, French Ministry of Foreign Affairs) and by the National Science Foundation through the Harvard MRSEC under award number DMR-0213805. This work was performed in part at the Center for Nanoscale Systems (CNS), a member of the National Nanotechnology Infrastructure Network (NNIN), which is supported by the National Science Foundation under NSF award no. ECS-0335765. CNS is part of the Faculty of Arts and Sciences at Harvard University.

\section{REFERENCES}

1. V. N. Manoharan, M. T. Elsesser and D. J. Pine, Science 301, 483-487 (2003).

2. $\quad$ W. Stöber, A. Fink and E. Bohn, J. Colloid Int. Sci. 26, 62-69 (1968).

3. C. P. Royall, M. E. Leunissen and A. van Blaaderen, J. Phys.: Condens. Matter, 15, S3581-S3596 (2003).

4. A. Perro, E. Duguet, O. Lambert, J.-C. Taveau, E. Bourgeat-Lami and S. Ravaine, Angew. Chem. Int. Ed., DOI: 10.1002/anie.200802562.

5. P. M. Johnson, C. M. van Kats and A. van Blaaderen, Langmuir, 21, 11510-11517 (2005).

6. http://physics.georgetown.edu/matlab/index.html - Matlab adaptation of IDL Particle Tracking software developed by David Grier, John Crocker and Eric Weeks. 